\title{
Solar system studies with the Indo-Belgian telescopes
}

\author{
Shashikiran Ganesh*, Aravind Krishnakumar, Kumar Venkataramani, \\ Archita Rai, Kiran Singh Baliyan, Umesh Chandra Joshi
}

Physical Research Laboratory, Navarangpura, Ahmedabad 380009, India

\begin{abstract}
Indian astronomers have been using their relatively modest instruments and telescopes to contribute greatly in understanding the minor bodies of the solar system. Here we discuss some of the results from observations using Indian facilities by researchers from India and abroad. Results are discussed in imaging/photometry, polarimetry and spectroscopy from the Indian telescopes up to 2 metres in aperture. It is hoped that access to the upcoming 2-m and 4-m class telescopes and sophisticated instrumentation would greatly improve our understanding with more exciting results coming up in future. Access to 2-m and 4-m class telescopes are one of the key points of the BINA collaboration. It is expected that our understanding of the solar system bodies, particularly the minor bodies and other small objects, would be greatly enhanced in terms of statistics as well as detailed characterization of individual ones.
\end{abstract}

Keywords: solar system - minor bodies - Indian observatories

\section{Introduction}

Indians have looked up at the skies and theorized about the planets and the solar system since ancient times ${ }^{1}$. In more recent times, astronomers working from Indian observatories have made original contributions to the general astronomical knowledge. One of the fundamental discoveries is the detection of Helium during a total Solar eclipse by the French astronomer Jules Janssen in Guntur, India (Emsley 2001).

In this paper we discuss some of the polarimetric, imaging and spectroscopic work, on solar system objects, carried out from the major Indian observatories. We caution the reader that this is not an exhaustive list of the contributions that can be found in the literature.

\section{Minor bodies of the solar system - observations from Indian facilities}

\subsection{Imaging / Photometry}

Indian observatories have been important contributors to worldwide campaigns on imaging and photometry of important cometary apparitions. E.g. Meech et al. (2017) observed the beginning of the

*e-mail: shashi@prl.res.in

${ }^{1}$ see https://en.wikipedia.org/wiki/Indian_astronomy for references and discussion 
activity of the long period comet C/2015 ER61 (PanSTARRS) by covering the heliocentric distance range of 8.9 to $4.8 \mathrm{AU}$ during the approach of the comet.

Vasundhara et al. (2017) present and discuss 23 events of the 2014-2015 mutual event series amongst the Galilean satellites. These are photometric and astrometric observations from the Vainu Bappu Observatory (VBO). Light curves of both eclipses and occultations are discussed. They show that with precise information (or models) of the intensity distribution of the eclipsed or occulted satellite disk a robust fit to the light curves is possible. Such a model intensity distribution should also explain the observed rotational curve of the satellites.

Joshi et al. (2011) observed comet C/2007 N3 (Lulin) between 2009, February 24 and 28. They carried out observations with the International Halley Watch blue and red continuum filters and the $\mathrm{R}$ broad band using the Optical Photo-polarimeter of the Physical Research Laboratory (PRL) on the 1.2-m telescope of Mount Abu Infra-Red Observatory (MIRO). They found a significant increase in brightness with decreasing phase angle in the range $10^{\circ}$ to $1.7^{\circ}$. The colour of the comet was found to be close to the solar colour showing that the grain size distribution was dominated by grains larger than $0.1 \mu \mathrm{m}$.

Comet 17P/Holmes underwent an outburst on 2007, October 24 about five months after perihelion passage. Observations were carried out by Joshi et al. (2010) in the period November-December 2007 using the PRL Optical Photo-polarimeter. Radial brightness variations were noticed over time in the red and blue continuum bands indicating a relative outward increase in the abundance of smaller dust particles.

Vasundhara \& Chakraborty (2004) obtained I and R band images of comet 81P/Wild 2 from the VBO. They explained the morphology of the fans in these images using models of the trajectories of the dust grains ejected from various locations on the comet.

\subsection{Polarimetry}

Continuum polarimetry of cometary comae provides insight into the dust grains of which they are constituted. Sunlight gets polarized due to scattering by cometary dust grains. The degree of polarization seen by the observer depends on the nature of the grains (specifically from their effect on the refractive index of the material) and the grain size distribution. It also varies with the wavelength of observation and the phase angle at the time of observation. Thus by having a good coverage in phase by observing the comet at different epochs in its orbit (e.g. Ganesh et al. 1998) and by carrying out the observations at different wavelengths, one may model the characteristics of the grains (see for e.g. Joshi et al. 1997). However, it is difficult to get a large coverage in phase angle. Hence, it is important to cover as many comets as possible since the main properties of the dust grains are rather similar. Variations are seen in the extent of polarization depending on the comet being dust-rich or gas-rich. Many Indian observatories have optical polarimeters and imaging polarimeters available for observations. In the paragraphs below, a few studies based on observations obtained with these instruments are summarized.

The ARIES imaging polarimeter (AIMPOL) has been used on the Sampurnanand 1.04-m telescope by Deb Roy et al. (2015) to observe comet C/2012 L2 (LINEAR) at a phase angle of $31.1^{\circ}$ before its perihelion passage. They used the R-band filter and observed the extended coma of the comet (covering a spatial range of $\sim 65000 \mathrm{~km}$ ). Variations are seen in intensity and polarization profile in different directions, consistent with the idea that the dust particles originate from active areas of the nucleus.

Imaging polarimetric observations of comet 67P/Churyumov-Gerasimenko were carried out during its 2008-2009 apparition by Hadamcik et al. (2010) using the Inter-University Centre for Astronomy and Astrophysics (IUCAA) imaging polarimeter on the IUCAA Girawali telescope. Ob- 
servations were carried out in December 2008 and April-May 2009 at phase angles of $36^{\circ}$ and $29^{\circ}$, respectively. Observations were also carried out at the Observatoire de Haute Provence (OHP; France) in March 2009. They found that the pre-perihelion polarization was similar to the one of other comets but an increase in polarization was seen post-perihelion. This indicates that an outburst occurred causing freshly ejected dust particles of sub-micron size. They also observed a strong seasonal effect related to the obliquity of the comet. From this they infer that the different grains originate from different hemispheres of the nucleus.

Ganesh et al. (2009) present polarization measurements of comet NEAT C/2001 Q4 in a large range of phase angles (85.6 to 55 $)$ observed in May and June of 2004. The observtions were done in the International Halley Watch (IHW) and BVR broad band filters with the PRL Optical Photopolarimeter on the 1.2-m MIRO telescope. In this phase angle range, the polarization increased with wavelength. They also mapped the polarization in a small aperture along a line crossing the coma in the direction of motion. A minor decrease in polarization was seen as the photocenter was crossed. They infer that the comet NEAT C/2001 Q4 belongs to the high polarization class of comets and that it has a typical grain size composition of a mixture of silicates and organics.

The work of Sen et al. (1990) on comet 1P/Halley is among the earliest imaging polarimetric observations of comets. The coma and tail region of the comet was observed on 1986, January 5 . They found a small region in the inner coma with polarization below $2 \%$. In the tail-ward direction, beyond the outer coma, two separate regions of enhanced polarization were identified with a polarization of more than $8 \%$. They conclude that the low polarization in the inner coma may be resulting from multiple scattering in a region of high dust concentration with probably recent dust jet ejecta.

\subsection{Spectroscopy}

Venkataramani et al. (2016) present low resolution spectra of comet C/2014 Q2 (Lovejoy) gathered in the period from January to May 2015, from perihelion passage to the post-perihelion phase. The spectra showed strong molecular emission bands $\left(\mathrm{C}_{2}, \mathrm{C}_{3}\right.$ and $\left.\mathrm{CN}\right)$ close to perihelion. They obtained scale-lengths for the molecules and also estimated the variation in gas and dust production rates as a function of heliocentric distance. They found the comet to be more active post-perihelion. A notable feature of this work is that it was done with a small $50-\mathrm{cm}$ telescope from Planewave Instruments with the low resolution spectrograph (LISA) from Shelyak Instruments at the MIRO of PRL.

The LISA spectrograph has also been used with the 1.2-m MIRO telescope with an appropriate focal reducer for several comets and asteroids since 2016. Some of the results have been presented at various conferences (see Venkataramani et al. 2018 a,b,c). In recent times, this instrument combination has been used to study the close passage of the comet 46P/Wirtanen as it approached and crossed perihelion. A sample spectrum from the observations is shown in Fig. 1. In the previous section, we have illustrated that a lot of polarimetric observations have been carried out in the $\mathrm{R}$ broad band to study cometary dust with the assumption that this band is relatively free from molecular emissions. However, as seen in the box marked in Fig.11, significant molecular emission is seen in the case of comet 46P/Wirtanen in this band. Hence, we recommend that polarimetric observations in the broad band filters (necessary in case of faint comets being observed with smaller telescopes) must be accompanied by spectroscopy at near simultaneous epochs. For bright comets, it would be very appropriate to use the narrow band continuum Hale-Bopp filters (Farnham \& Schleicher 2000) or similar equivalents for polarimetric studies of cometary dust. 


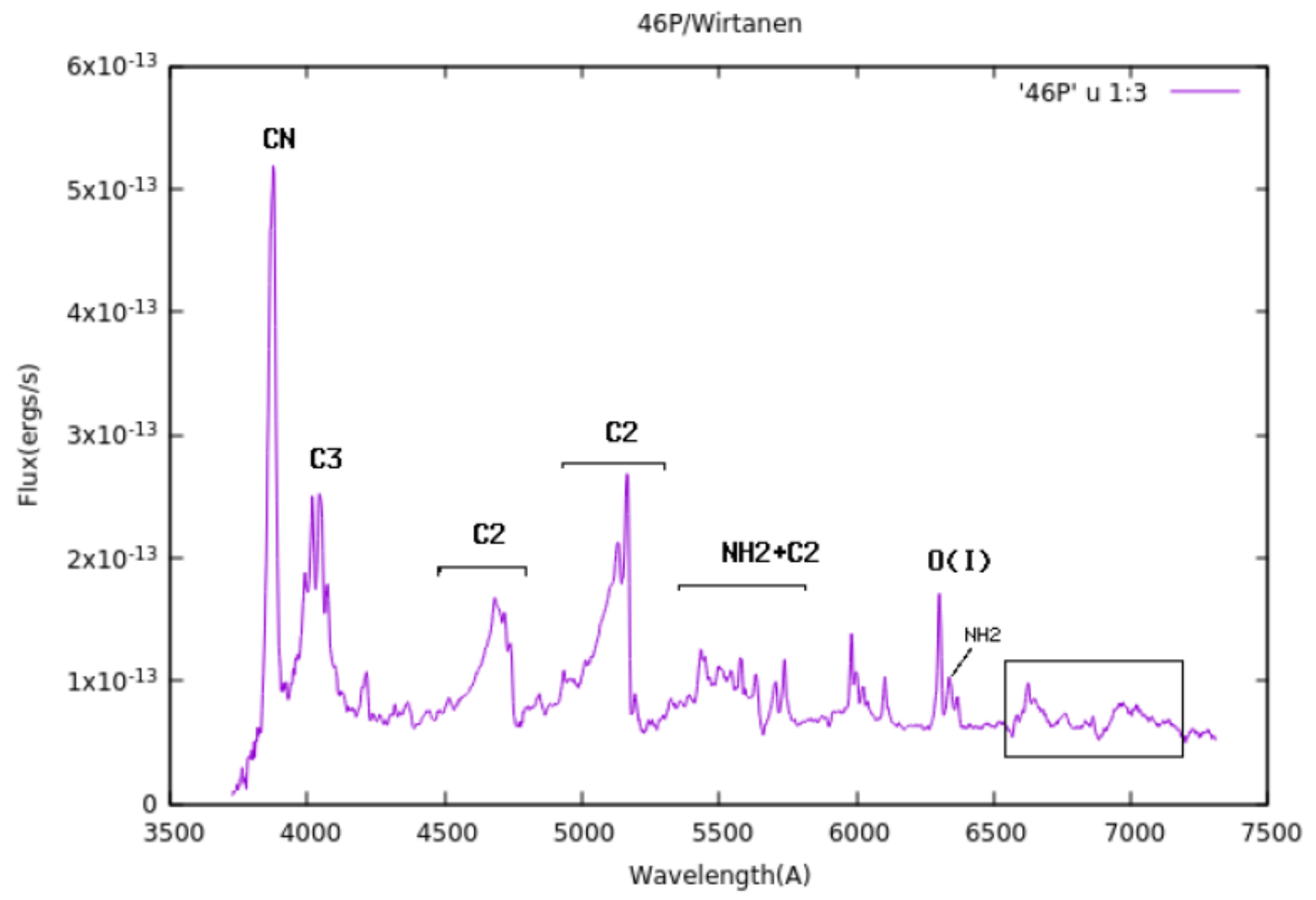

Figure 1: Spectrum of comet 46P/Wirtanen from the MIRO with the LISA spectrograph. The rectangular box shows features at wavelengths traditionally considered to be free of molecular emission in cometary spectra.

\section{Prospects for future science using Indian facilities}

The availability of larger telescopes with the corresponding availability of new-genaration instruments will allow for more exciting times in solar system research using Indian facilities. The availability of optical and infrared spectroscopic instruments on the Indo-Belgian 3.6-m Devasthal Optical Telescope (DOT) and on the upcoming 2.5-m PRL telescope (also being manufactured by AMOS, Belgium) will have a large contribution in the further understanding of the minor bodies of the solar system. We refer to the the papers in the instrumentation section of these proceedings to get an overview of the new facilities being created.

\section{Summary}

In this contribution, we have briefly covered some of the most important results in imaging, spectroscopy and polarimetry of primarily comets to illustrate the importance of the current Indian and upcoming Indo-Belgian observing facilities for solar system research. 


\section{Acknowledgements}

We acknowledge the grant from Department of Science and Technology (India) and the Belgian Federal Science Policy Office (Belgium) which enabled the first author to travel to Brussels and to participate in the 2nd BINA conference. Work at PRL is funded by the Department of Space, Govt. of India.

\section{References}

Deb Roy P., Das H. S., Medhi B. J. 2015, Icar, 245, 241

Emsley J. 2001, Nature's Building Blocks, Oxford University Press, Oxford

Farnham T. L., Schleicher D. G. 2000, Icar, 147, 180

Ganesh S., Joshi U. C., Baliyan K. S., Deshpande M. R. 1998, A\&AS, 129, 489

Ganesh S., Joshi U. C., Baliyan K. S. 2009, Icar, 201, 666

Hadamcik E., Sen A. K., Levasseur-Regourd A. C., Gupta R., Lasue J. 2010, A\&A, 517, A86

Joshi U. C., Baliyan K. S., Ganesh S. et al. 1997, A\&A, 319, 694

Joshi U. C., Ganesh S., Baliyan K. S. 2010, MNRAS, 402, 2744

Joshi U. C., Ganesh S., Baliyan K. S. 2011, MNRAS, 412, L58

Meech K. J., Schambeau C. A., Sorli K. et al. 2017, AJ, 153, 206

Sen A. K., Joshi U. C., Deshpande M. R., Debi Prasad C. 1990, Icar, 86, 248

Vasundhara R., Chakraborty P. 2004, ApJ, 616, 1278

Vasundhara R., Selvakumar G., Anbazhagan P., 2017, MNRAS, 468, 501

Venkataramani K., Ghetiya S., Ganesh S. et al. 2016, MNRAS, 463, 2137

Venkataramani K., Ganesh S., 2018a, EPSC, 12, 1207

Venkataramani K., Ganesh S. 2018b, EPSC, 12, 1220

Venkataramani K., Ganesh S., Joshi U. C. 2018c, 42nd COSPAR Scientific Assembly, Pasadena, id: B1.1-55-18 